\title{
Self-Awareness-Based Resource Allocation Strategy for Containment of Epidemic Spreading
}

\author{
Xiaolong Chen, ${ }^{1}$ Quanhui Liu, ${ }^{2}$ Ruijie Wang $\mathbb{D}^{3},{ }^{3}$ Qing Li, ${ }^{1}$ and Wei Wang $\mathbb{D D}^{4}$ \\ ${ }^{1}$ Financial Intelligence and Financial Engineering Key Laboratory of Sichuan Province, School of Economic Information \\ Engineering, Southwestern University of Finance and Economics, Chengdu 611130, China \\ ${ }^{2}$ College of Computer Science, Sichuan University, Chengdu 610065, China \\ ${ }^{3}$ Aba Teachers University, Aba 623002, China \\ ${ }^{4}$ Cybersecurity Research Institute, Sichuan University, Chengdu 610065, China
}

Correspondence should be addressed to Ruijie Wang; ruijiewang001@163.com

Received 7 February 2020; Revised 26 April 2020; Accepted 9 May 2020; Published 23 May 2020

Academic Editor: Mahdi Jalili

Copyright ( $) 2020$ Xiaolong Chen et al. This is an open access article distributed under the Creative Commons Attribution License, which permits unrestricted use, distribution, and reproduction in any medium, provided the original work is properly cited.

Resource support between individuals is of particular importance in controlling or mitigating epidemic spreading, especially during pandemics. However, there remains the question of how we can protect ourselves from being infected while helping others by donating resources in fighting against the epidemic. To answer the question, we propose a novel resource allocation model by considering the awareness of self-protection of individuals. In the model, a tuning parameter is introduced to quantify the reaction strength of individuals when they are aware of the disease. And then, a coupled model of resource allocation and disease spreading is proposed to study the impact of self-awareness on resource allocation and its impact on the dynamics of epidemic spreading. Through theoretical analysis and extensive Monte Carlo simulations, we find that in the stationary state, the system converges to two states: the whole healthy or the completely infected, which indicates an abrupt increase in the prevalence when there is a shortage of resources. More importantly, we find that too cautious and too selfless for the people during the outbreak of an epidemic are both not suitable for disease control. Through extensive simulations, we locate the optimal point, at which there is a maximum value of the epidemic threshold, and an outbreak can be delayed to the greatest extent. At last, we study further the effects of the network structure on the coupled dynamics. We find that the degree heterogeneity promotes the outbreak of disease, and the network structure does not alter the optimal phenomenon in behavior response. Based on the results of this study, a constructive suggestion is that in the face of a global pandemic, individuals or countries should strengthen mutual support and cooperation while doing their own prevention to suppress the epidemic optimally.

\section{Introduction}

Controlling the outbreak of epidemic is one of the most important topics in human history. During the past decades, the onset of several major global health threats such as the 2003 spread of SARS, the H1N1 influenza pandemic in 2009, and the western Africa Ebola outbreaks in 2014 have deprived tens of thousands of lives all around the world [1-3]. In 2019, a novel coronavirus causing severe acute respiratory disease (COVID-19) emerged. By the end of April 2020, there have been more than two million confirmed COVID19 infections reported all over the world [4]. The surge in infections has led to a severe shortage of medical resources.
Thousands of confirmed and suspected cases await treatment [5]. Facing the rapid outbreak of the disease, the contribution of resources from healthy individuals is necessary, but the self-protection of the susceptible individuals cannot be ignored. Thus, the immediate problem is how can we protect ourselves from being infected while helping others in fighting against the epidemic.

A large number of researchers from various disciplines have made efforts to study the topic of optimal resource allocation in disease suppressing in the past years [6-10]. For example, Preciado et al. [11] studied the problem of the optimal distribution of vaccination resources to control epidemic spreading based on complex networks. They found 
the cost-optimal distribution of the vaccination resource when different levels of vaccination are allowed through a convex framework. Further, they studied the optimal allocation of the preventive and corrective resources to achieve the highest level of containment when the budget is given in advance or finding the minimum budget required when the budget is not specified [12]. Chen et al. [13] solved the problem of optimal allocation of a limited medical resource based on mean-field theory. The above works considered the problem from a mathematical perspective, and were solved from the premise that both the number of resources and the spreading state of the epidemic are fixed.

However, the real scenario is more complicated than a static mathematical problem. Multiple dynamical processes always interact and coevolve $[14,15]$, forming a more realistic starting point. For example, during the outbreak of an epidemic, the quantity of available resources is largely affected by the propagation of the disease, and in turn, the dynamical change of the necessary resources influences the dynamical property of the epidemic spreading. The coevolution of multiple dynamical processes has attracted extensive research in recent years [16]. Böttcher [17] studied the coevolution of resource and epidemics, and they found a critical recovery cost that if the cost is above the critical value, epidemics spiral out of control into "explosive" spread. Chen et al. [18] studied the effects of social support from local connections on the spreading dynamics of the epidemic. They proposed a coevolution spreading model on multiplex networks and found a hybrid phase transition on networks with heterogeneous degree distribution. In this multiplex network framework, Chen et al. [19] further studied the impact of preferential resource allocation on the social subnetwork on the spreading dynamics of the epidemic.

In addition to the physical resources that can directly mitigate or control the epidemic spreading, the awareness of the epidemic in populations is another type of resource. The public can perceive the threat of the epidemic social network through a platform and mass media and then take measures to protect themselves. Therefore, the interplay between awareness diffusion and epidemic spreading is another topic that have attracted extensive research [20-22]. A mass of works addressed the problem from different perspectives considering, for example, the risk perception and behavioral changes [23-25]. Granell et al. [14] studied the interplay between the processes of epidemic spreading and awareness diffusion on top of multiplex networks and found a metacritical. Wang et al. [26] studied the coevolution mechanisms using both real online and offline data. Based on the empirical analysis, they proposed a coupled model on a multiplex network to study the coupled dynamics of these two processes.

Although a mass of works on the coevolution of awareness diffusion and disease spreading have been carried out, a question that needs to be addressed is how does awareness affect the behavior of resource donation in populations. To answer this question, a novel resource allocation model that incorporates the influence of the awareness is proposed in this paper. We further consider that the healthy individuals in an outbreak of disease are providers of essential resources, and they can not only produce resources, but also donate them to those who need. The donation behavior in turn leads to less resource for selfprotection and a more significant probability to be infected. Thus, when the healthy individuals are aware of the threat of the disease and refuse to donate resources, they would have more resources for self-protection and a lower probability of being infected; however, this is not conducive to the overall prevention and control of the disease. Thus, we are faced with a game-like problem between resource contribution and self-protection [27]. To solve the problem optimally, we should study the complex dynamic mechanisms among selfawareness, resource donation, and epidemic spreading. Thus, a coupled dynamical model on top of the complex networks is proposed, and a dynamic message-passing method is adopted for the theoretical analysis in this paper.

Based on the model, we first investigate the influence of awareness on the coupled dynamics of resource allocation and disease spreading on scale-free networks. To quantify the awareness for self-protection, a parameter $\alpha$ is assigned to each individual. Through theoretical analysis and numeric simulations, we find that the system has only two stationary states, namely, the absorb state and the globe outbreak state. With the increase of $\alpha$, the epidemic threshold first increases and then decreases, which indicates that there is an optimal value of $\alpha$. Further, we locate the optimal value $\alpha_{\text {opt }}$ through extensive simulations, under which the disease can be suppressed to the greatest extent. Then, we explain qualitatively the optimal phenomenon. And then, we investigate the impact of degree heterogeneity on the coupled dynamics and find that the degree heterogeneity does not alter the optimal phenomenon and the abrupt increase in prevalence with a shortage of resource. At last, we find that the epidemic threshold increases with the decrease in degree heterogeneity, which suggests that network heterogeneity promotes the outbreak of disease.

\section{Model Description}

2.1. Epidemic Model. A resource-based epidemiological susceptible-infected-susceptible model (r-SIS) is proposed to describe the coupled dynamics of epidemic spreading and resource allocation on a complex network. Individuals are represented by nodes in the network, and an adjacency matrix $A$ is introduced to represent the connection between nodes. If there is an edge between nodes $i$ and $j$, the element $a_{i j}=1$, otherwise $a_{i j}=0$. According to this scheme, any individual can be in two different states: susceptible (S) and infected (I). The infection propagates between each pair of I-state and S-state neighbors with an infection rate $\widetilde{\lambda}$ in one contact, which is assumed to depend on whether the S-state nodes donate resources, see the details in Section 2.2. At any time $t$, each I-state node $i$ recovers with a recovery rate $r_{i}(t)$. Resources including medical, funds, and food can promote recovery of patients from disease $[28,29]$. Thus, we define the recovery rate of each I-state node as a function of the resource quantity received from healthy neighbors in this paper. As each I-state node may get a different amount of resource, the recovery rate varies from node to node. Consequently, the recovery rate of any node $i$ at time $t$ can be defined as 


$$
r_{i}(t)=1-(1-\mu)^{\varepsilon \omega_{i}(t)},
$$

where $\omega_{i}(t)$ is the resource quantity of node $i$ received from healthy neighbors at time $t$ and $\mu$ is the basic recovery rate. A parameter $\varepsilon \in[0,1]$ is introduced in our model to represent the resource utilization rate [30]. Since in the real scenario, there is the common phenomenon of the waste on resource $[31,32]$ in medical and other service systems, implying the resource received from healthy neighbors may not be fully utilized on curing and recovery. Without loss of generality, we set $\epsilon=0.6$ throughout this work, i.e., only $60 \%$ of the resources received are used.

For the r-SIS model, the value $\rho_{i}(t)$ is defined as the probability that any node $i$ is in the infected state. The fraction of infected nodes in a network of size $N$ at time $t$ can be calculated by averaging the overall $N$ nodes:

$$
\rho(t)=\frac{1}{N} \sum_{i=1}^{N} \rho_{i}(t) .
$$

Further, we define the prevalence of the disease in the stationary state as $\rho \equiv \rho(\infty)$.

\subsection{Resource Allocation Model Based on Behavior Response.} In the real scenario, healthy individuals can produce resources. For simplicity, we consider that each individual (node) in the network can generate one unit resource at a time step. During an outbreak of a disease, the susceptible individuals can perceive the threat of the disease intuitively by acquiring the information from neighbors. Generally, the more infected neighbors of an individual, the deeper he/she is aware of the disease $[33,34]$. People aware of the disease may have different reactions [33]. Also, to quantify the reaction strength of an individual to the local information of disease, a tuning parameter $\alpha$ is introduced. Based on the description above, we can define the probability that a healthy individual with $m$ infected neighbors donates resource as

$$
q(m)=q_{0}(1-\alpha)^{m}
$$

where $q_{0}$ is a basic donation probability. When $\alpha=0$, all healthy nodes have the same donation probability $q_{0}$. Besides, we consider that a healthy node can donate one unit resource equally to its I-state neighbors at a time. Based on the resource allocation scheme, the amount of resource $\omega_{j \longrightarrow i}$ that node $j$ with $m$ infected neighbors donate to one of its I-state neighbor, $i$, can be expressed as

$$
\omega_{j \longrightarrow i}=q(m) \frac{1}{m} .
$$

When disease breaks out in the human population, people aware of the disease in their proximity can take measures to reduce their susceptibility, leading to a reduction in the effective rate of infection $[14,35]$. We consider that if an individual is aware and refuses to donate resource for self-protection, it reduces its infectivity by a factor $c$. The basic infection rate is denoted as $\lambda$, and the annealing infection rate is denoted as $\tilde{\lambda}$, which can thus be expressed as

$$
\tilde{\lambda}= \begin{cases}\lambda, & \text { if distribute the resources, } \\ c \lambda, & \text { else. }\end{cases}
$$

If a healthy individual donates resource to infected neighbors, the individual has a larger probability to be infected; on the contrary, if the individual does not donate resource, there is a relatively smaller probability to be infected. The annealing infection rate of any node $i$ can also be expressed as a function of $q$ :

$$
\tilde{\lambda}_{i}=q(m) \lambda+[1-q(m)] c \lambda .
$$

\section{Dynamic Message-Passing Method}

In order to theoretically analyze the dynamic processes, we develop a generated dynamic message-passing method (GDMP) [36, 37]. In this method, the message $\theta_{j \longrightarrow i}$ is defined on the directed edges of a network to carry causal information of the flow of contagion, which can only transfer one way along directed links. $\theta_{j \longrightarrow i}$ represents the probability that node $j$ is infectious because it was infected by one of its neighbors other than node $i$. In computing $\theta_{j \longrightarrow i}$, we only take into account the contributions to $\rho_{j}$ that come from the neighbors other than $i$. The higher order process of $j$ being infected by $i$ and then passes the infection back to $i$ is neglected. Combining $\theta_{j \rightarrow i}$ and equation (3) for resource allocations, the resources $\omega_{i}(t)$ that an infected node $i$ receive from its healthy neighbors can be expressed as

$$
\omega_{i}(t)=\sum_{j} a_{i j}\left[1-\theta_{j \longrightarrow i}(t)\right] \frac{q\left[m_{j}(t)\right]}{m_{j}(t)},
$$

where $m_{j}(t)$ is the expected number of I-state neighbors of node $j$ at time $t$, which is expressed as

$$
m_{j}(t)=\sum_{h \neq i} a_{j h} \theta_{h \longrightarrow j}(t)+1
$$

where the plus one takes into account that node $i$ is infected at this moment. The factor $\left(1-\theta_{j \longrightarrow i}(t)\right)$ in equation (7) stands for the probability that node $j$ is susceptible at time $t$. With the definition above, the discrete-time version of the evolution of $\rho_{i}(t)$ in a time interval $\Delta t$ reads [38]

$$
\rho_{i}(t+\Delta t)=\left(1-\rho_{i}(t)\right)\left(1-\Omega_{i}(t)\right)+\left[1-r_{i}(t) \Delta t\right] \rho_{i}(t),
$$

where $\Omega_{i}(t)$ is the probability that the node is not infected by any neighbor with the product being over the set $\mathcal{N}_{i}$ of the neighbors of node $i$. The expression of $\Omega_{i}(t)$ is as follows:

$$
\Omega_{i}(t)=\prod_{j \in \mathcal{N}_{i}}\left[1-\Delta t \tilde{\lambda}_{i}(t) \theta_{j \longrightarrow i}(t)\right] .
$$

Note that the first term on the right-hand side of equation (9) stands for the probability that node $i$ is in S-state and infected by at least one of its neighbors. The second term is the probability that node $i$ is in I-state and 
does not recover. Similarly, we can get the time evolution of $\theta_{j \longrightarrow i}(t)$ as

$\theta_{j \longrightarrow i}(t+\Delta t)=\left(1-\theta_{j \longrightarrow i}(t)\right)\left(1-\phi_{j \longrightarrow i}(t)\right)+\left(1-r_{j}(t) \Delta t\right) \theta_{j \longrightarrow i}(t)$,

where $\phi_{j \longrightarrow i}(t)$ is the probability that node $j$ is not infected by any of its neighbors excluding node $i$, which can be expressed as

$$
\phi_{j \longrightarrow i}(t)=\prod_{\ell \in \mathscr{N}_{j} \backslash i}\left[1-\Delta t \tilde{\lambda}_{j}(t) \theta_{\ell \longrightarrow j}(t)\right] .
$$

The product in equation (12) is over the set $\mathcal{N}_{j} \backslash i$ of the neighbors of $j$ excluding $i$. Further, by setting $\Delta t=1$ and considering situation in a stationary state, equations (9) and (11) become

$$
\begin{aligned}
\rho_{i} & =\left(1-\rho_{i}\right)\left(1-\Omega_{i}\right)+\left(1-r_{i}\right) \rho_{i}, \\
\theta_{j \longrightarrow i} & =\left(1-\theta_{j \longrightarrow i}\right)\left(1-\phi_{j \longrightarrow i}\right)+\left(1-r_{j}\right) \theta_{j \longrightarrow i} .
\end{aligned}
$$

Through numerical iteration, we can compute the infection probability of any node at any time $\rho_{i}(t)$ and prevalence $\rho$ in the stationary state for different values of $\alpha$ and $\lambda$. However, the equations can only be solved numerically, except for the trivial solutions of $\rho_{i}=0$ and $\theta_{j \longrightarrow i}=0$, for all $i=1, \ldots, N$, which leads to an overall $\rho=0$ phase of an all-healthy population.

Due to nonlinearities in equations (7)-(12), they do not have a closed analytic form, and this disallows obtaining the epidemic threshold $\lambda_{c}$ for fixed values of $\alpha$, such that $\rho>0$ if $\lambda>\lambda_{c}$ and $\rho=0$ when $\lambda<\lambda_{c}$. The calculation of $\lambda_{c}$ can be performed by considering that when $\lambda \longrightarrow \lambda_{c}, \rho_{i} \longrightarrow 0$ and $\theta_{j \longrightarrow i} \longrightarrow 0$, and the number of infected neighbors of any healthy node is approximately zero in the thermodynamic limit. Then, prior to reaching $\lambda_{c}$, the expression $\left(1-\theta_{j \longrightarrow i}\right) \longrightarrow 1$ is valid. We can get a physical picture that the isolated infected nodes are well separated and surrounded by healthy nodes, and any infected node $i$ can receive all the resource from each of its neighbors. By adding these assumptions to equation (7), resource $\omega_{i}$ becomes $\omega_{i}=k_{i} q_{0}(1-\alpha)$. By linearizing equation (1) and neglecting the second-order terms for small $\mu$, we obtain

$$
\begin{aligned}
r_{i}(t) & \approx \epsilon \mu \omega_{i}(t) \\
& =\epsilon \mu k_{i} q_{0}(1-\alpha) .
\end{aligned}
$$

Equation (15) suggests that the recovery rate is proportional to the node degree and inversely proportional to $\alpha$ when $\lambda \longrightarrow \lambda_{c}$. For the sake of clarity, the basic recovery rate is set at $\mu=0.01$ in this paper. Further equations (10) and (12) can also be linearized using $\theta_{j \longrightarrow i} \approx 0$ as

$$
\begin{aligned}
q_{i} & \approx 1-\tilde{\lambda}_{i} \sum_{j=1}^{N} a_{j i} \theta_{j \longrightarrow i}, \\
\phi_{j \longrightarrow i} & \approx 1-\tilde{\lambda}_{j} \sum_{l \longrightarrow h \in V_{E}} \mathbf{M}_{j \longrightarrow i, l \longrightarrow h} \theta_{l \longrightarrow h},
\end{aligned}
$$

where $V_{E}$ is the set of directed edges and $\mathbf{M}$ is the $\left|V_{E}\right| \times\left|V_{E}\right|$ nonbacktracking matrix [39] of the network with the elements labelled by the edges:

$$
\mathbf{M}_{j \longrightarrow i, l \longrightarrow h}=\delta_{j h}\left(1-\delta_{i l}\right) \text {, }
$$

with $\delta_{i l}$ being the Dirac delta function. Substituting equation (17) into equation (14) and ignoring the higher order terms of $\theta_{j \longrightarrow i}$ give

$$
\sum\left(-\delta_{l j} \delta_{i h} r_{j}+\tilde{\lambda}_{j} \mathbf{M}_{j \longrightarrow i, l \longrightarrow h}\right) \theta_{l \longrightarrow h}=0 .
$$

Finally, considering that $m_{j}=1$ when $\lambda \longrightarrow \lambda_{c}$, equation (6) becomes

$$
\widehat{\lambda} \equiv \tilde{\lambda}_{j}=\left[(1-c) q_{0}(1-\alpha)+c\right] \lambda .
$$

To estimate the epidemic threshold, we calculate the average recovery rate as

$$
\langle r\rangle=\epsilon \mu\langle k\rangle q_{0}(1-\alpha)
$$

By inserting equations (20) and (21) into equation (19), we get

$$
\sum\left(-\delta_{l j} \delta_{i h}\langle r\rangle+\hat{\lambda} \mathbf{M}_{j \longrightarrow i, l \longrightarrow h}\right) \theta_{l \longrightarrow h}=0 .
$$

The system of equations in equation (22) has a nontrivial solution if and only if $\langle r\rangle / \widehat{\lambda}$ is an eigenvalue of the matrix $M$ [38]. The lowest value $\hat{\lambda}_{c}$ is then given by

$$
\hat{\lambda}_{c}=\frac{\langle r\rangle}{\Lambda_{\max }},
$$

where $\Lambda_{\max }$ is the largest eigenvalue of $\mathbf{M}[15,37,40]$.

\section{Numerical Verification and Simulation Results}

In this section, we study systematically the effects of selfawareness and the network structure on the coupled dynamics of resource allocation and disease spreading, respectively, through numerical verification and Monte Carlo simulations. In the simulation, the synchronous updating method [41, 42] is applied to the disease infection and resource allocation processes. Within each time increment $\Delta t$, where $\Delta t=1$ in this paper, infection propagates from any I-state node $j$ to S-state node $i$ with probability $\widetilde{\lambda}_{i} \Delta t$ and any I-state node $j$ recovers to $S$-state with a probability $r_{j} \Delta t$. With the spreading of disease, the resource allocation process co-occurs. The dynamics terminate once it enters a steady state in which the number of infected nodes only fluctuates within a small range. Note that, we fix the factor $c$ at a constant value $c=0.05$ throughout the paper, such that if any healthy individual $j$ chooses to reserve their resource, the probability that they are infected in one contact with an infected neighbor reduces to $\widetilde{\lambda}_{j}=0.05 \lambda$.

4.1. Effects of Self-Awareness on the Spreading Dynamics. In this section, we investigate the effects of awareness for self-protection on the spreading dynamics. We consider that 
the coupled processes of resource allocation and disease spreading takes place on a scale-free network, as many realworld networks have skewed degree distributions [43-46]. To build the network, we adopt the uncorrelated configuration model (UCM) $[47,48]$, according to a given degree distribution $P(k) \sim k^{-\gamma}$ with maximum degree $k_{\max }=\sqrt{N}$ [49] and minimum degree $k_{\min }=3$, which assures no degree correlation of the network when $N$ is sufficiently large. To avoid the influence of the network structure on the result, the degree exponent is set at $\gamma=2.4$, the network size is set at $N=10000$, and the average degree is set at $\langle k\rangle=8$ in the simulations. In addition, we leverage the susceptibility measure $\chi$ to determine the epidemic threshold through simulations [50], which is expressed as

$$
\chi=N \frac{\left\langle\rho^{2}\right\rangle-\langle\rho\rangle^{2}}{\langle\rho\rangle},
$$

where $\langle\ldots\rangle$ represents the ensemble average over all realizations. The epidemic threshold can then be determined when the value of $\chi$ exhibits diverging peaks at the certain infection rate $[50,51]$.

We first investigate the effects of self-awareness on the spreading dynamics using Monte Carlo simulations. Initially, a fraction of $\rho(0)=0.1$ nodes are selected randomly as seeds, and the remaining nodes are in the susceptible state. To present different reaction strength of individuals when they are aware of a certain disease from local information, we select eight typical values of $\alpha$ from $\alpha=0.1$ to $\alpha=0.9$ in the simulation. In Figures 1(a) and 1(c), we plot the prevalence $\rho$ in the stationary state as a function of basic infection rate $\lambda$ for different $\alpha$. Symbols in Figures 1(a) and 1(c) represent the results obtained by Monte Carlo simulations, and lines are the theoretical results obtained from numeric iterations, respectively. From the curves in Figures 1(a) and 1(c), we observe that the system converges to two possible stationary states: either the whole population is healthy, or it becomes completely infected for any $\alpha$, which tells us that when there is a shortage in resource, the disease breaks out abruptly.

Besides, we can observe from Figures 1(a) and 1(b) that with the increase of $\alpha$ from $\alpha=0.1$ to $\alpha=0.5$, the epidemic threshold increases gradually, see the peaks of $\chi$ for the corresponding $\alpha$. It reveals that the stronger the individual's sense of self-protection, the more delayed the outbreak of the disease within this parameter interval (see the right arrow). On the contrary, we observe from Figures 1(c) and 1(d) that when $\alpha$ increases from $\alpha=0.6$ to $\alpha=0.9$, the threshold decreases gradually, which reveals that the disease breaks out more easily with a stronger sense of self-protection within this parameter interval (see the left arrow). The phenomenon suggests that too cautious or too selfless for the people during the outbreak of an epidemic are both not suitable for disease control, and there is an optimal value of the reaction strength, at which an epidemic outbreak is postponed to the greatest extent.

We further study systematically the effects of behavior response and the basic infection rate on the spreading dynamics. In Figure 2, we exhibit the full phase diagram ( $\alpha-$ $\lambda$ ) of the coupled dynamics of resource allocation and disease spreading. Colors in Figure 2(a) encode the fraction of infected nodes $\rho$ in the stationary state. The epidemic threshold $\lambda_{c}$, marked by red circles, rises monotonically until it reaches the maximum at $\alpha_{\text {opt }}$ (indicated by the blue dotted line) and then falls gradually with the increase of $\alpha$. Besides, we observe that there are only two possible stationary states: the whole healthy (marked by blue color) and the whole infected of the population (marked by yellow color).

Figure 3(a) plots the time evolution of $\rho(t)$ for six typical values of $\alpha$ when the basic infection rate is fixed at $\lambda=0.04$. We find that when the value of $\alpha$ is small, the system converges to a stationary state rapidly, such as $\rho(\infty)=1.0$ for $\alpha=0.1$. With the increase of $\alpha$, it takes a longer time for the system to reach a stationary state. Further, to exhibit the effects of $\alpha$ on the dynamics more intuitively, we plot the fraction of infected nodes at a fixed time $t=200$ as a function of $\alpha$ in Figure 3(b), which is denoted as $\rho(\alpha)$ for the sake of clarity. We observe that the value of $\rho(\alpha)$ decreases continuously with $\alpha$ until reaching the minimum value at $\alpha_{\mathrm{opt}} \approx 0.48$ (marked by red circle in Figure 3(b)) and then increases gradually with $\alpha$.

Next, we qualitatively explain the optimal phenomena by studying the time evolution of the critical quantities.

We begin by studying the case when $\alpha$ is small, for example, $\alpha=0.1$. We observe in Figure 4 that in the initial stage, the donation probability for $\alpha=0.1$ is the highest (see the blue line in the top panel of Figure 4(a)) since a smaller value of $\alpha$ means a higher willingness of healthy individuals to allocate resources. Although the resource of healthy individuals can improve the recovery probability of infected neighbors to a certain extent, it also makes themselves more likely to be infected. We can observe in Figures 4(b) and 4(c) that the average recovery rate $\langle r\rangle$ and the infection rate $\langle\tilde{\lambda}\rangle$ is the highest for $\alpha=0.1$; meanwhile, there is a lowest value of the effective infection rate $\langle\widetilde{\lambda}\rangle /\langle r\rangle$, as shown in Figure $4(\mathrm{~d})$. However, with the high probability of being infected for the healthy nodes, the number of infected individuals increases at a high rate (see the blue line in the bottom pane of Figure 4(a)). When people are aware of the increment of the infected neighbors, they reduce their donation willingness, which leads to a reduction in infection rate $\langle\tilde{\lambda}\rangle$, as shown in Figures 4(a) and 4(b). Consequently, with less resource received from healthy neighbors, the recovery rate of infected nodes reduces accordingly, see Figure $4(\mathrm{c})$, which leads to an increase of the effective infection rate $\langle\tilde{\lambda}\rangle /\langle r\rangle[15]$. The increase in $\langle\tilde{\lambda}\rangle /\langle r\rangle$ has led to a further increase in the number of infected nodes. Then, people become more aware of the threat of disease and thus reduce the probability of resource donation further, which leads to a further decrease in the infection rate $\langle\tilde{\lambda}\rangle$ and the recovery rate $\langle r\rangle$ and finally the increase of the effective infection rate $\langle\tilde{\lambda}\rangle /\langle r\rangle$.

Specifically, we observe from Figure 4(d) that when it surpasses a critical time $t^{*}$, indicated by the dotted line in the figure, the value of $\langle\widetilde{\lambda}\rangle /\langle r\rangle$ proliferates, which suggests that in this stage, the infection of healthy individuals is much faster than the recovery of infected individuals. With more newly infected nodes, the donation probability $\langle q\rangle$ and the infection rate $\langle\widetilde{\lambda}\rangle$ decrease further, which results in less 


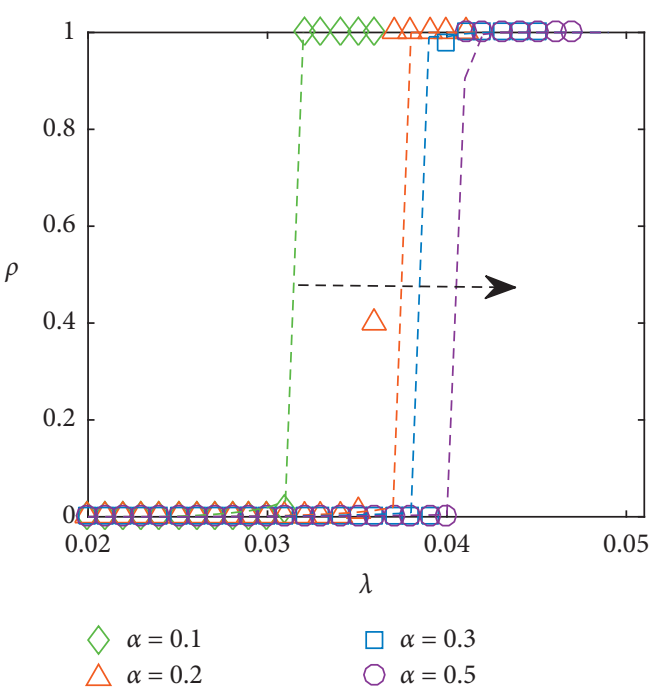

(a)

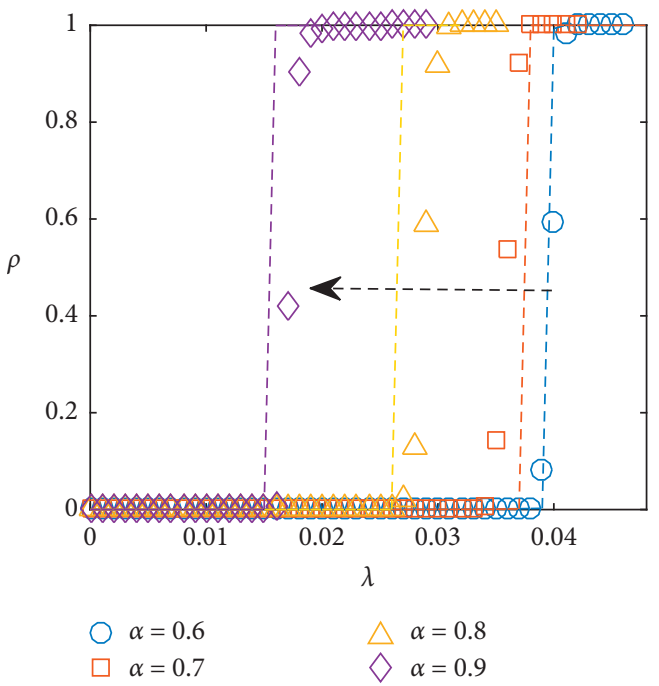

(c)

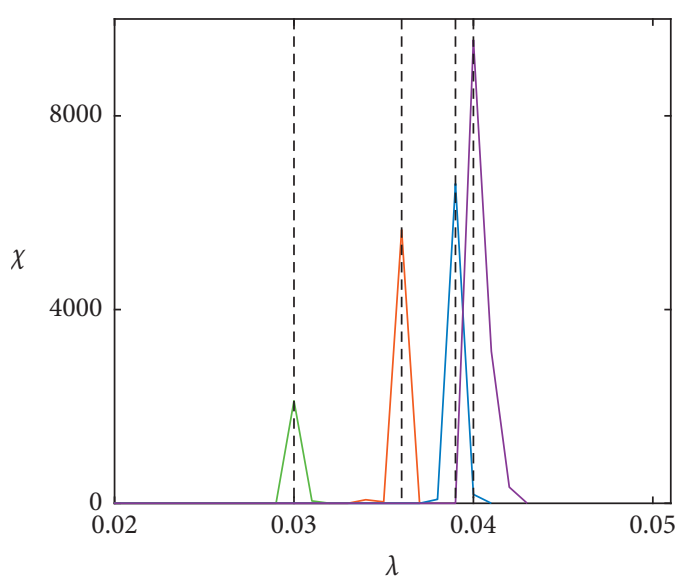

(b)

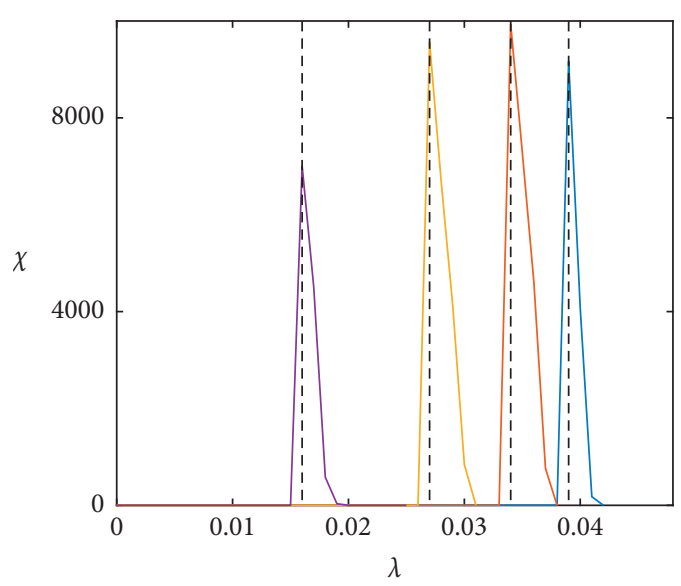

(d)

Figure 1: Effects of self-awareness on the dynamics of disease spreading on a scale-free network. (a) and (c) The prevalence $\rho$ in the stationary state as a function of basic infection rate $\lambda$ for varieties of reaction strength $\alpha$. Symbols represent the results obtained from Monte Carlo simulations, and lines represent the results of the GDMP method. (b) and (d) The corresponding susceptibility measure $\chi$ as a function of $\lambda$. Data are obtained by averaging over 500 independent simulations.

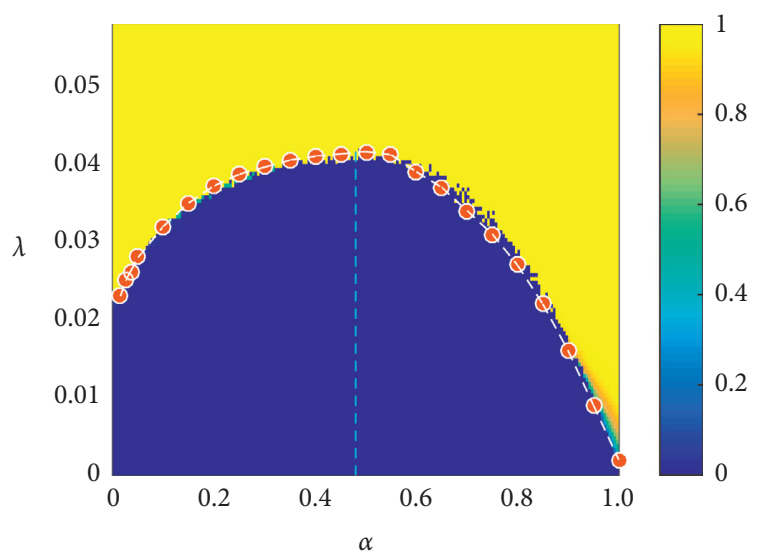

Figure 2: The phase diagram in the parameter plane $(\alpha-\lambda)$ on a scale-free network. Colors encode the value of $\rho$ obtained from Monte Carlo simulations. Red circles connected by dotted lines represent theoretical predictions of epidemic threshold $\lambda_{c}$. The blue dotted line indicates the location of optimal value $\alpha_{\text {opt }}$. Data are obtained by averaging 50 Monte Carlo simulations for each point in the grid $200 \times 200$. 


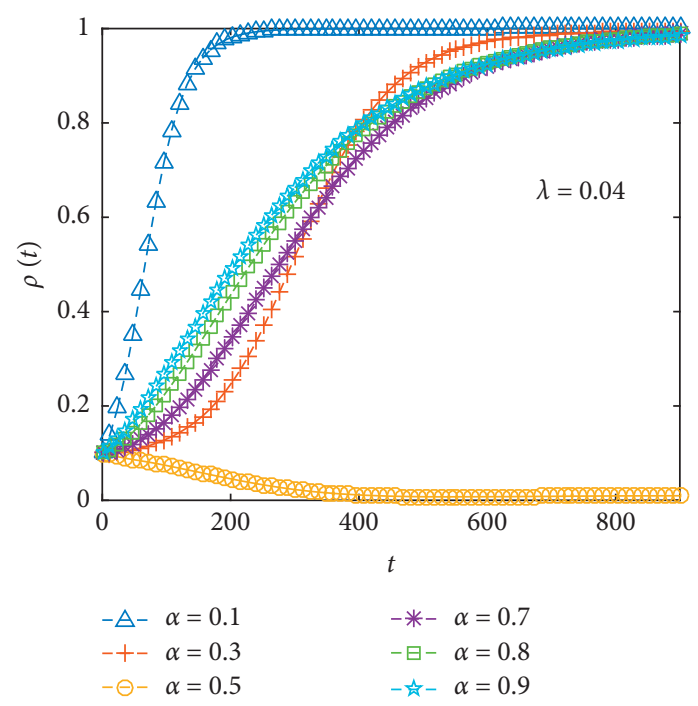

(a)

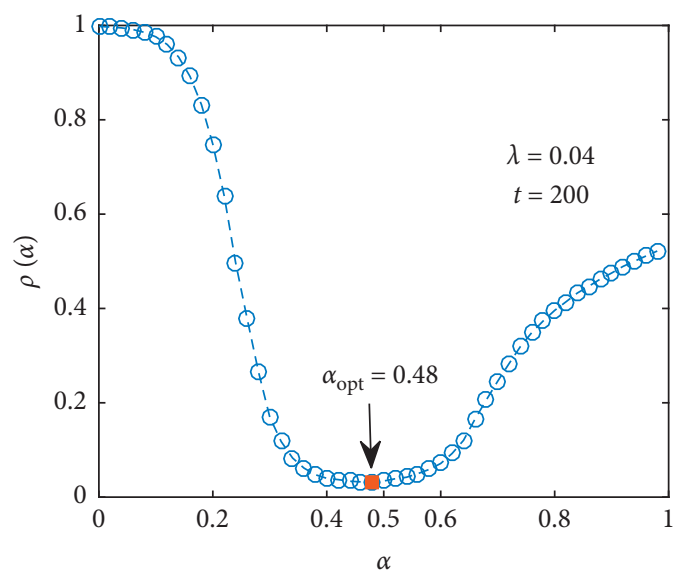

(b)

Figure 3: Effects of behavior response on evolution of the fraction of infected nodes $\rho(t)$. (a) The time evolution of $\rho(t)$ for varieties of $\alpha$ using Monte Carlo simulations for a fixed value of $\lambda=0.04$. (b) Plot of the fraction of infected nodes versus the change in $\alpha$ at a fixed time $t=200$ and infection rate $\lambda=0.04$. The results of the simulations are obtained by averaging over 300 realizations.

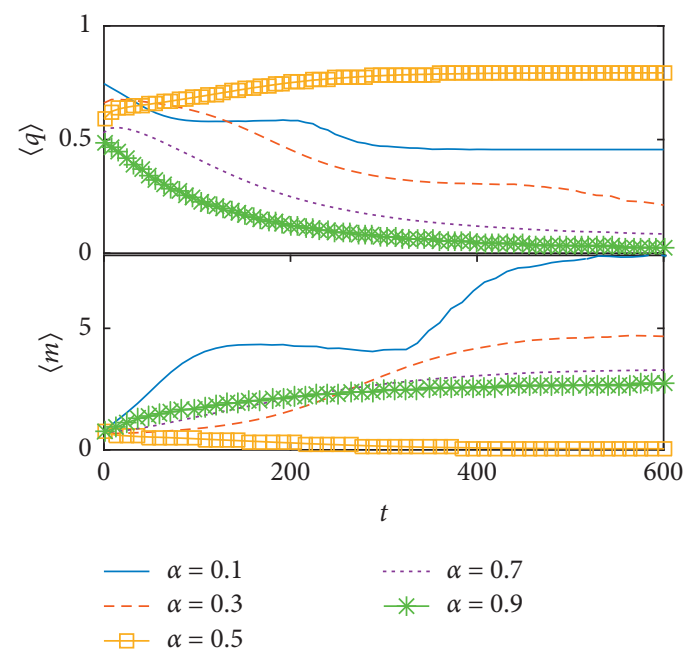

(a)

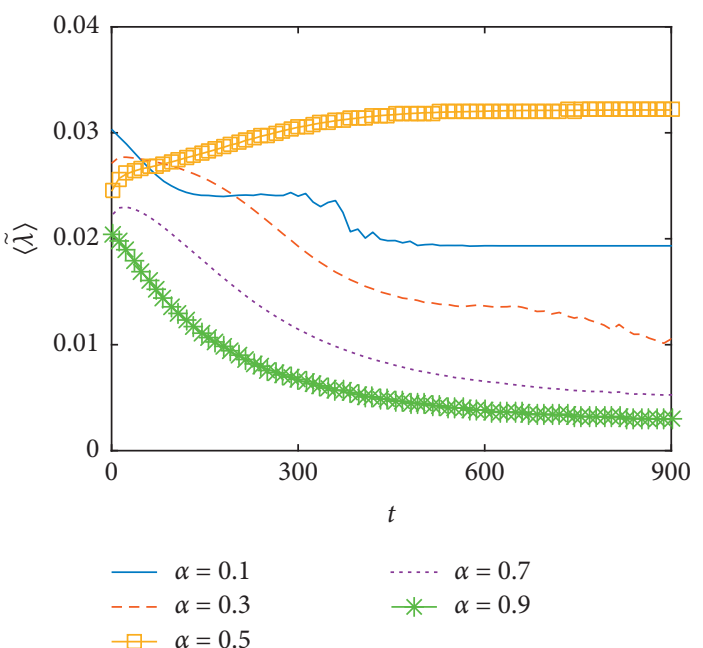

(b)

Figure 4: Continued. 


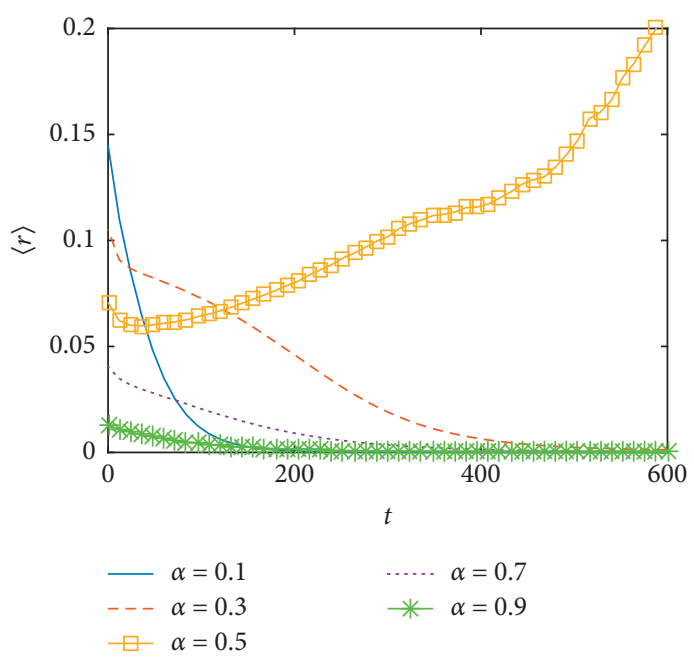

(c)

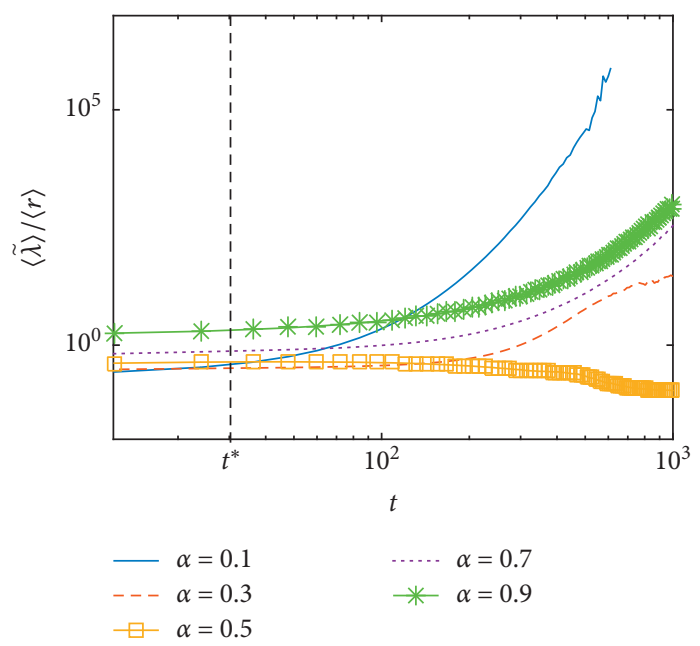

(d)

Figure 4: Plots of the critical parameters versus $t$ for typical values of $\alpha$. (a) Top pane: time evolution of the average donation rate $\langle q\rangle$. Bottom pane: the evolution of average number of infected neighbors of all nodes $\langle m\rangle$. (b) Time evolution of the average infection rate $\langle\lambda\rangle$. (c) The complete evolution of the average recovery rate $\langle r\rangle$. (d) Log-log plots of the average effective infection rate $\langle\tilde{\lambda}\rangle /\langle r\rangle$. Basic infection rate is fixed at $\lambda=0.04$. The results of the simulations are obtained by averaging over 300 realizations.

resources donated to support the recovery of infected nodes. Thus, the recovery rate of infected nodes $\langle r\rangle$ drops abruptly, which in turn promotes the increases of the effective infection rate $\langle\tilde{\lambda}\rangle /\langle r\rangle$ further, and then more and number of infected nodes appear. Consequently, the cascading failure of the entire system occurs.

Based on the above analysis for a small value of $\alpha$, i.e., $\alpha=0.1$, we can reasonably explain why people are more willing to contribute a resource while the disease is more likely to break out.

Secondly, we study the case when $\alpha$ is significant, for example, $\alpha=0.9$. As a larger value of $\alpha$ means more sensitive of the individuals to the disease and a lower willingness to allocate resources. Thus, we observe from Figure 4(a) that, initially, there is a smallest value of $\langle q\rangle$ (see the green stars in top pane of Figure $4(\mathrm{a}))$ and the infection rate $\langle\bar{\lambda}\rangle$. Consequently, the infected nodes receive the lowest value of the resource to recover, which leads to the smallest value of the recovery rate $\langle r\rangle$, as shown by the green stars in Figure 4(c). Then, the recovery of infected nodes is delayed leading to a high effective infection rate. We can observe in Figure 4(d) that, when $\alpha=0.9$, there is a highest value of $\langle\tilde{\lambda}\rangle /\langle r\rangle$. The high effective infection rate leads to a rapid increase in the number of infected nodes. We can observe in the bottom pane of Figure 4(a) that, in the early stage, there is a second largest value of $\langle m\rangle$ for $\alpha=0.9$, as denoted by the green stars. The large value of $\langle m\rangle$ can further reduce the willingness of resource donation for the healthy individuals; thus, we can observe a continuous decline in $\langle q\rangle$ and $\langle\widetilde{\lambda}\rangle$. The worse thing is that the recovery rate of infected nodes keeps declining with less and less resource (see the curve in Figure 4(c)), which leads to a rapid growth of $\langle\widetilde{\lambda}\rangle /\langle r\rangle$ (see the curve in Figure 4(d)).

Thus, we can explain the reason why a higher sense of self-protection of the population cannot suppress the disease effectively.
At last, we observe in Figure 4 that, when the value of $\alpha$ is around the optimal value $\alpha_{\text {opt }}$, there is a relatively lower value of $\langle q\rangle$ comparing to the case of $\alpha=0.1$ in the initial stage, which results in a lower value of $\langle\tilde{\lambda}\rangle$ (see the yellow squares in Figures 4(a) and 4(b)). The lower willingness of resource donation induces to a relatively smaller value of the recovery rate $\langle r\rangle$, as shown in Figure 4(c). However, we can observe from Figure 4(d) that the effective infection rate $\langle\widetilde{\lambda}\rangle /\langle r\rangle$ keeps the lowest value in the early stage, which suggests that the disease propagates slowly in the population, and the number of infected nodes increases slowly, which is verified by the curve in the bottom pane of Figure 4(a). Further, the small value of $\langle m\rangle$ promotes the increase of $\langle q\rangle$ (see the curve in the top pane of Figure 4(a)), which results in the increase of the recovery rate $\langle r\rangle$. And finally, the effective infection rate $\langle\tilde{\lambda}\rangle /\langle r\rangle$ decreases further, as shown in Figure $4(\mathrm{~d})$. Thus, the disease can be suppressed to the greatest extend.

Through the three steps, we explain the optimal phenomena in the coupled dynamics of resource allocation and disease spreading.

Finally, we further verify our explanation by studying the critical quantities as the function of parameter $\alpha$ at a fixed time $t$ and basic infection rate $\lambda$. Figures 5(a) to 5(d) plot the values of $\langle q\rangle,\langle m\rangle,\langle\widetilde{\lambda}\rangle,\langle r\rangle$, and $\langle\widetilde{\lambda}\rangle \mid\langle r\rangle$ as a function of $\alpha$ when $t=200$ and $\lambda=0.04$. For the sake of clarity, we denote the local minimum and maximum value as $X_{\mathrm{Lmin}}$ and $X_{\max }$ and the global minimum and maximum value as $X_{\min }$ and $X_{\max }$, respectively, where $X \in[\langle q\rangle,\langle m\rangle,\langle\tilde{\lambda}\rangle,\langle\tilde{\lambda}\rangle /\langle r\rangle]$. We observe that although when $\alpha$ is around $\alpha_{\text {opt }}$, there is a local maximum of $\langle q\rangle_{\operatorname{Lmax}}$ and $\langle\widetilde{\lambda}\rangle_{\mathrm{Lmax}}$. The recovery rate reaches maximum $\langle q\rangle_{\max }$, and the effective infection rate reaches the lowest $(\langle\tilde{\lambda}\rangle /\langle r\rangle)_{\min }$, which indicates that the disease can be optimally suppressed at this point. 


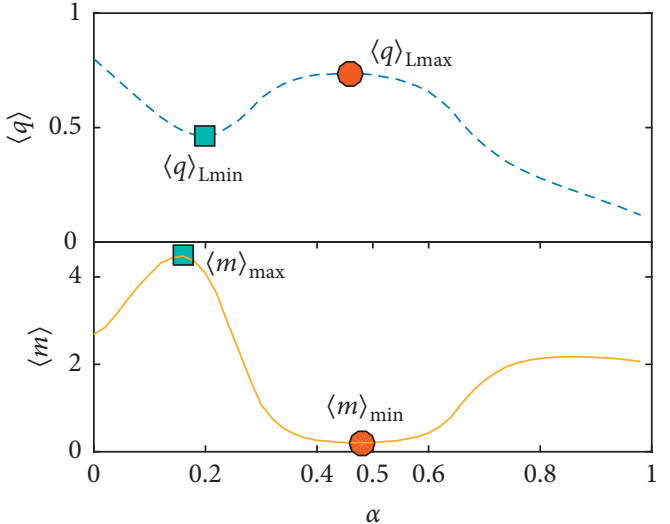

(a)

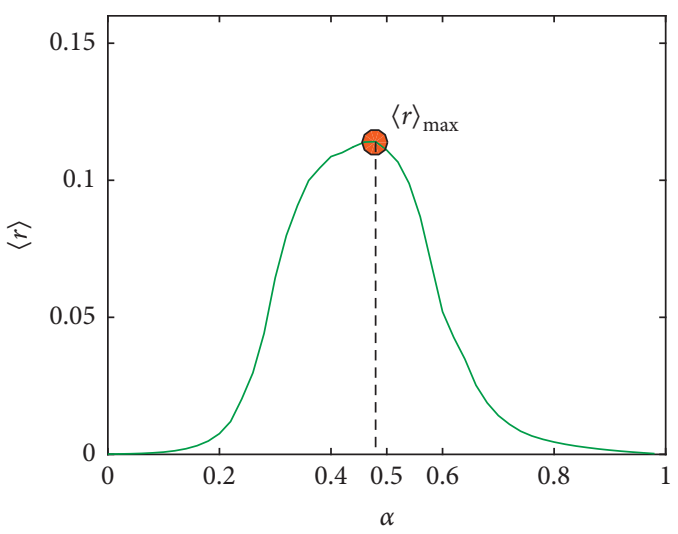

(c)

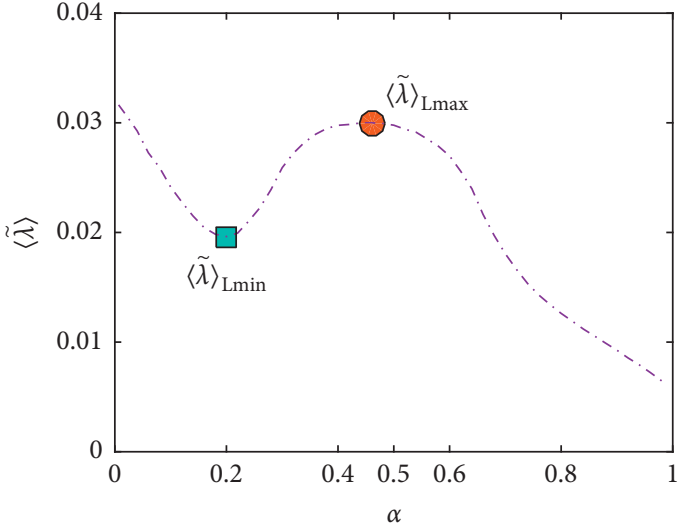

(b)

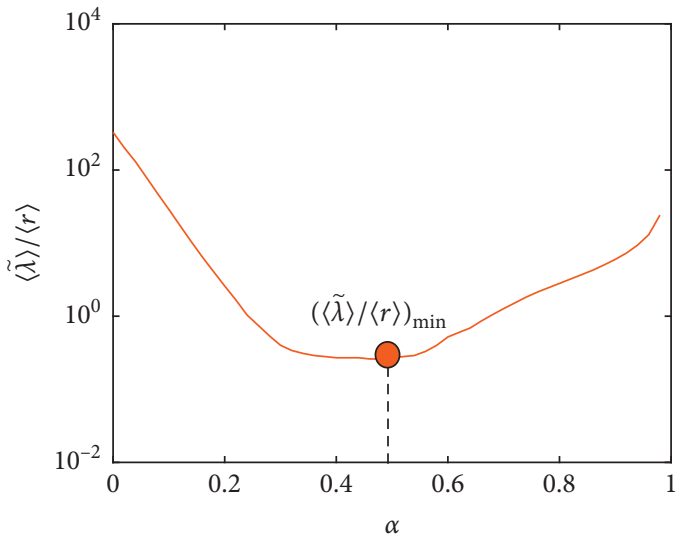

(d)

Figure 5: Plots of the critical parameters versus $\alpha$ at fixed time $t=200$ and basic infection rate $\lambda=0.04$. (a) Top pane: the average donation rate $\langle q\rangle$ as a function of $\alpha$. Bottom pane: the average number of infected neighbors of all nodes $\langle m\rangle$ as a function of $\alpha$. (b) The average infection rate $\langle\widetilde{\lambda}\rangle$ as a function of $\alpha$. (c) The average recovery rate $\langle r\rangle$ as a function of $\alpha$. (d) Plots of average effective infection rate $\langle\widetilde{\lambda}\rangle /\langle r\rangle$ as a function of $\alpha$. The results of the simulations are obtained by averaging over 300 realizations.

\subsection{Effects of Network Structure on Spreading Dynamics.} In this section, we investigate the effects of the network structure on the coupled dynamics of resource allocation and disease spreading. To avoid the impact of reaction strength on the result, the parameter $\alpha$ is fixed at $\alpha=0.5$. In addition, we adopt the UCM model to generate scalefree networks with different degree distributions $P(k) \sim k^{-\gamma}$. As the degree heterogeneity decreases with the increase of the power exponent $\gamma[52,53]$, it approaches to random regular networks (RRNs) when $\gamma \longrightarrow \infty[18]$.

Figure 6 plots the prevalence $\rho$ in the stationary state as a function of the basic infection rate $\gamma$ for networks with four typical values of $\gamma: \gamma=2.4$ (blue circles), $\gamma=2.8$ (upper triangles), $\gamma=3.2$ (purple squares), and $\gamma \longrightarrow \infty$ (red rhombus). We observe that there are only two stationary states of the system: all healthy or completely infected for all networks, which implies that the network structure does not alter the first-order transition of $\rho$. Besides, we find that, with an increase of $\gamma$, the outbreak of disease is delayed gradually. It suggests that the degree heterogeneity enhances the disease spreading, which is consistent with the existing research conclusions [54].

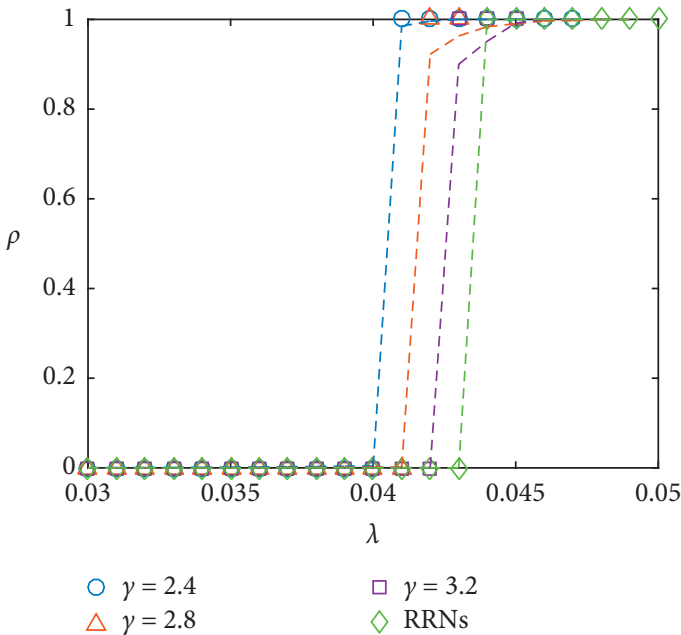

FIGURE 6: The prevalence $\rho$ in the stationary as a function of $\lambda$ on scale-free networks with degree exponent $\gamma=2.4$ (blue circles), $\gamma=2.8$, and $\gamma=3.2$ (purple squares). And, the result on random regular networks (RRNs) marked by the red rhombus. Symbols represent the results obtained from Monte Carlo simulations, and lines represent results of the GDMP method. The parameter $\alpha$ is fixed at $\alpha=0.5$. 


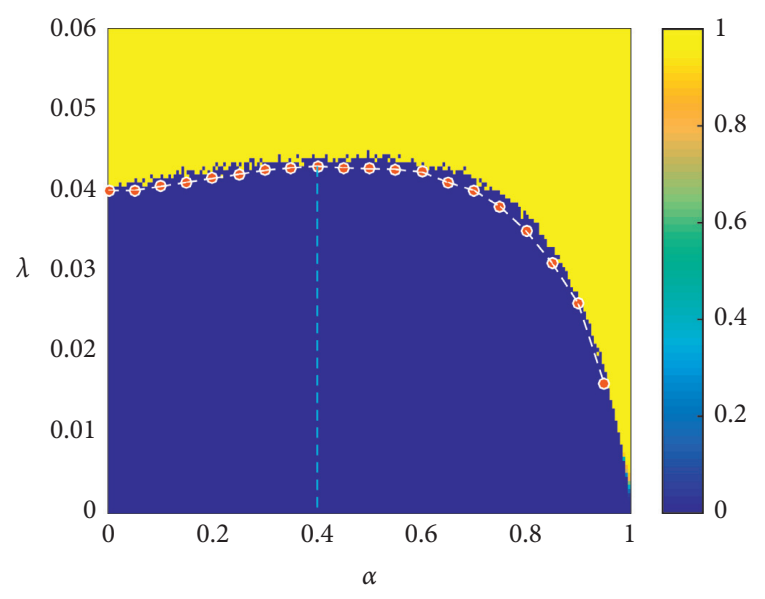

FIGURE 7: The phase diagram in the parameter plane $(\alpha-\lambda)$ on RRNs. Colors encode the value of $\rho$ obtained from Monte Carlo simulations. Red circles connected by dotted lines represent theoretical predictions of epidemic threshold $\lambda_{c}$. The blue dotted line indicates the location of optimal value $\alpha_{\text {opt }}$. Data are obtained by averaging 50 Monte Carlo simulations for each point in the grid $200 \times 200$.

In the end, we study the effects of behavior response on the spreading dynamics systematically. Figure 7 is the phase diagram in the parameter plane $(\alpha-\lambda)$ on RRNs. Colors encode the prevalence in the stationary state $\rho$. We find that there is also an optimal value $\alpha_{\text {opt }}$, at which the epidemic threshold reaches the maximum, indicated by the blue dotted line in Figure 7. The results suggest that the network structure does not alert the optimal phenomenon in behavior response.

\section{Discussion}

In this paper, we have focused on the problem of how can we protect ourselves from being infected while helping others by donating resources during an outbreak of an epidemic. To answer this question, we have proposed a novel resource allocation model in controlling the epidemic spreading by considering the following two facts, namely, the healthy individuals are the providers of essential resources and there is a kind of game between individual's self-protection and resource contribution. To quantify the awareness for selfprotection, a parameter $\alpha$ has been assigned to each individuals in the model. Besides, to study the coupled dynamics of resource allocation and disease spreading, a resourcebased SIS model has been proposed. First of all, we have theoretically analyzed the model by using a generated dynamic message-passing method and then carried out extensive Monte Carlo simulations on both scale-free and random regular networks. Through theoretical analysis and simulations, we have found that the coupled dynamics converges to two stationary states: the whole infected or all healthy, which indicates that a shortage of resource can induce an abrupt outbreak of the epidemic. More importantly, we have found that too cautious or too selfless for the people during the outbreak of an epidemic are both not suitable for epidemic containment. There is an optimal (balance) point where the epidemic spreading can be controlled to the greatest extent. It also suggests that one can donate resource appropriately to support the people in need, but at the same time, they should reserve the right amount of resources for self-protection. Further, we have located the optimal point. At last, we have investigated the effects of the network structure on the coupled dynamics and found that the degree heterogeneity promotes the outbreak of disease, and the network structure does not alter the optimal phenomenon in behavior response.

Our research is of practical significance in the context of the global outbreak of COVID-19. It will guide us to make the most reasonable choice between resource contribution and self-protection when perceiving the threat of disease and also have a direct application in the development of strategies to suppress the outbreaks of epidemics. Moreover, our suggestions that in the face of a global pandemic, individuals or countries should strengthen mutual support and cooperation while doing their own prevention are consistent with the current measures taken by most individuals and countries in combating the epidemic. At present, not only the individuals but also the nations are donating resources to support each other while ensuring its own prevention and control needs. For example, when the outbreak in China is effectively contained, it announces assistance to many COVID-19 countries by donating medical resources, such as respirator, mask, nucleic acid testing reagent, and sending the medical staffs [55].

There is still much more work need to be done. For example, the SIS model adopted in this work has its own limitations, and it cannot fully describe the characteristics of most real epidemics. As we all know that there is an incubation period in COVID-19, so an SEIR model may be more suitable. Moreover, in some other epidemics, the recovered individuals would obtain a short acquired immune and then turn into the susceptible state again, so the SIRS model is more suitable. Therefore, the research of the dynamical properties when the present mechanisms are applied in various epidemic models would be the future directions.

\section{Data Availability}

The data used to support the findings of this study are available from the corresponding author upon request.

\section{Conflicts of Interest}

The authors declare that they have no conflicts of interest.

\section{Acknowledgments}

This work was supported by the Fundamental Research Funds for the Central Universities (nos. JBK190972, JBK171113, and JBK170505), National Natural Science Foundation of China (nos. 61903266, 71671141, 71873108, and 61703292), the Financial Intelligence \& Financial Engineering Key Lab of Sichuan Province, China Postdoctoral 
Science Foundation (no. 2018M631073), and China Postdoctoral Science Special Foundation (no. 2019T120829).

\section{References}

[1] K. H. Chan, P. H. Li, S. Y. Tan, Q. Chang, and J. P. Xie, "Epidemiology and cause of severe acute respiratory syndrome (sars) in Guangdong, People's Republic of China, in February, 2003," Lancet, vol. 362, no. 9393, pp. 1353-1358, 2003.

[2] M. P. Girard, J. S. Tam, O. M. Assossou, and M. P. Kieny, "The 2009 a (H1N1) influenza virus pandemic: a review," Vaccine, vol. 28, no. 31, pp. 4895-4902, 2010.

[3] WHO Ebola Response Team, "Ebola virus disease in west africathe first 9 months of the epidemic and forward projections," New England Journal of Medicine, vol. 371, no. 16, pp. 1481-1495, 2014.

[4] World Health Organization, Coronavirus Disease 2019 (Covid-19) Situation Report-96, WHO Geneva, Switzerland, 2020, https:// www.who.int/emergencies/diseases/novel-coronavirus-2019/ situation-reports.

[5] R. Li, S. Pei, B. Chen et al., "Substantial undocumented infection facilitates the rapid dissemination of novel coronavirus (sars-cov2)," Science, vol. 368, no. 6490, pp. 489-493, 2020.

[6] Y. Wan, S. Roy, and A. Saberi, "Designing spatially heterogeneous strategies for control of virus spread," IET Systems Biology, vol. 2, no. 4, pp. 184-201, 2008.

[7] E. Gourdin, J. Omic, and P. Van Mieghem, "Optimization of network protection against virus spread," in Proceedings of the 2011 8th International Workshop on the Design of Reliable Communication Networks (DRCN), pp. 86-93, IEEE, Krakow, Poland, 2011.

[8] A. Y. Lokhov and D. Saad, "Optimal deployment of resources for maximizing impact in spreading processes," Proceedings of the National Academy of Sciences, vol. 114, no. 39, pp. E8138-E8146, 2017.

[9] D. Zhao, L. Wang, Z. Wang, and G. Xiao, "Virus propagation and patch distribution in multiplex networks: modeling, analysis, and optimal allocation," IEEE Transactions on Information Forensics and Security, vol. 14, no. 7, pp. 1755-1767, 2019.

[10] S. Li, D. Zhao, X. Wu, Z. Tian, A. Li, and Z. Wang, "Functional immunization of networks based on message passing," Applied Mathematics and Computation, vol. 366, Article ID 124728, 2020.

[11] V. M. Preciado, M. Zargham, C. Enyioha, A. Jadbabaie, and G. Pappas, "Optimal vaccine allocation to control epidemic outbreaks in arbitrary networks," in Proceedings of the 52nd IEEE Conference on Decision and Control, IEEE, Firenze, Italy, pp. 7486-7491, December 2013.

[12] V. M. Preciado, M. Zargham, C. Enyioha, A. Jadbabaie, and G. J. Pappas, "Optimal resource allocation for network protection against spreading processes," IEEE Transactions on Control of Network Systems, vol. 1, no. 1, pp. 99-108, 2014.

[13] H. Chen, G. Li, H. Zhang, and Z. Hou, "Optimal allocation of resources for suppressing epidemic spreading on networks," Physical Review E, vol. 96, no. 1, Article ID 012321, 2017.

[14] C. Granell, S. Gómez, and A. Arenas, "Dynamical interplay between awareness and epidemic spreading in multiplex networks," Physical Review Letters, vol. 111, no. 12, Article ID 128701, 2013.
[15] R. Pastor-Satorras, C. Castellano, P. Van Mieghem, and A. Vespignani, "Epidemic processes in complex networks," Reviews of Modern Physics, vol. 87, no. 3, pp. 925-979, 2015.

[16] W. Wang, Q.-H. Liu, J. Liang, Y. Hu, and T. Zhou, "Coevolution spreading in complex networks," Physics Reports, vol. 820, no. 2, pp. 1-51, 2019.

[17] L. Böttcher, O. Woolley-Meza, N. A. Araújo, H. J. Herrmann, and D. Helbing, "Disease-induced resource constraints can trigger explosive epidemics," Scientific Reports, vol. 5, Article ID 16571, 2015.

[18] X. Chen, R. Wang, M. Tang, S. Cai, H. E. Stanley, and L. A. Braunstein, "Suppressing epidemic spreading in multiplex networks with social-support," New Journal of Physics, vol. 20, no. 1, Article ID 013007, 2018.

[19] X. Chen, W. Wang, S. Cai, H. E. Stanley, and L. A. Braunstein, "Optimal resource diffusion for suppressing disease spreading in multiplex networks," Journal of Statistical Mechanics: Theory and Experiment, vol. 2018, no. 5, Article ID 053501, 2018.

[20] P. Hu, L. Ding, and X. An, "Epidemic spreading with awareness diffusion on activity-driven networks," Physical Review E, vol. 98, no. 6, Article ID 062322, 2018.

[21] P. Zhu, X. Wang, S. Li, Y. Guo, and Z. Wang, "Investigation of epidemic spreading process on multiplex networks by incorporating fatal properties," Applied Mathematics and Computation, vol. 359, pp. 512-524, 2019.

[22] Z. Wang, Q. Guo, S. Sun, and C. Xia, "The impact of awareness diffusion on sir-like epidemics in multiplex networks," Applied Mathematics and Computation, vol. 349, pp. 134-147, 2019.

[23] S. Funk, E. Gilad, and V. A. A. Jansen, "Endemic disease, awareness, and local behavioural response," Journal of Theoretical Biology, vol. 264, no. 2, pp. 501-509, 2010.

[24] Q. Wu, X. Fu, M. Small, and X.-J. Xu, "The impact of awareness on epidemic spreading in networks," Chaos: An Interdisciplinary Journal of Nonlinear Science, vol. 22, no. 1, Article ID 013101, 2012.

[25] H. Yang, C. Gu, M. Tang, S.-M. Cai, and Y.-C. Lai, "Suppression of epidemic spreading in time-varying multiplex networks," Applied Mathematical Modelling, vol. 75, pp. 806-818, 2019.

[26] W. Wang, Q.-H. Liu, S.-M. Cai, M. Tang, L. A. Braunstein, and H. E. Stanley, "Suppressing disease spreading by using information diffusion on multiplex networks," Scientific Reports, vol. 6, no. 1, Article ID 29259, 2016.

[27] H.-F. Zhang, Z. Yang, Z.-X. Wu, B.-H. Wang, and T. Zhou, "Braess's paradox in epidemic game: better condition results in less payoff," Scientific Reports, vol. 3, no. 1, pp. 1-8, 2013.

[28] J. A. Kulik and H. I. Mahler, "Social support and recovery from surgery," Health Psychology, vol. 8, no. 2, pp. 221-238, 1989.

[29] B. Nausheen, Y. Gidron, R. Peveler, and R. Moss-Morris, "Social support and cancer progression: a systematic review," Journal of Psychosomatic Research, vol. 67, no. 5, pp. 403-415, 2009.

[30] A. S. Mackie, L. Pilote, R. Ionescu-Ittu, E. Rahme, and A. J. Marelli, "Health care resource utilization in adults with congenital heart disease," The American Journal of Cardiology, vol. 99, no. 6, pp. 839-843, 2007.

[31] T. Jaarsma, R. Halfens, H. Huijer Abu-Saad et al., "Effects of education and support on self-care and resource utilization in patients with heart failure," European Heart Journal, vol. 20, no. 9, pp. 673-682, 1999.

[32] M. Gul and A. F. Guneri, "A computer simulation model to reduce patient length of stay and to improve resource 
utilization rate in an emergency department service system," International Journal of Industrial Engineering, vol. 19, no. 5, pp. 221-231, 2012.

[33] S. Funk, M. Salathé, and V. A. A. Jansen, "Modelling the influence of human behaviour on the spread of infectious diseases: a review," Journal of the Royal Society Interface, vol. 7, no. 50, pp. 1247-1256, 2010.

[34] W. Wang, M. Tang, H.-F. Zhang, and Y.-C. Lai, "Dynamics of social contagions with memory of nonredundant information," Physical Review E, vol. 92, no. 1, Article ID 012820, 2015.

[35] S. Funk, E. Gilad, C. Watkins, and V. A. A. Jansen, "The spread of awareness and its impact on epidemic outbreaks," Proceedings of the National Academy of Sciences, vol. 106, no. 16, pp. 6872-6877, 2009.

[36] B. Karrer and M. E. Newman, "Message passing approach for general epidemic models," Physical Review E, vol. 82, no. 1, Article ID 016101, 2010.

[37] M. Shrestha, S. V. Scarpino, and C. Moore, "Message-passing approach for recurrent-state epidemic models on networks," Physical Review E, vol. 92, no. 2, Article ID 022821, 2015.

[38] S. Gómez, A. Arenas, J. Borge-Holthoefer, S. Meloni, and Y. Moreno, "Discrete-time Markov chain approach to contact-based disease spreading in complex networks," Europhysics Letters, vol. 89, no. 3, Article ID 38009, 2010.

[39] F. Krzakala, C. Moore, E. Mossel et al., "Spectral redemption in clustering sparse networks," Proceedings of the National Academy of Sciences, vol. 110, no. 52, pp. 20935-20940, 2013.

[40] W. Wang, M. Tang, H. Eugene Stanley, and L. A. Braunstein, "Unification of theoretical approaches for epidemic spreading on complex networks," Reports on Progress in Physics, vol. 80, no. 3, Article ID 036603, 2017.

[41] B. Schönfisch and A. de Roos, "Synchronous and asynchronous updating in cellular automata," BioSystems, vol. 51, no. 3, pp. 123-143, 1999.

[42] W. Wang, P. Shu, Y.-X. Zhu, M. Tang, and Y.-C. Zhang, "Dynamics of social contagions with limited contact capacity," Chaos: An Interdisciplinary Journal of Nonlinear Science, vol. 25, no. 10, Article ID 103102, 2015.

[43] M. Girvan and M. E. J. Newman, "Community structure in social and biological networks," Proceedings of the National Academy of Sciences, vol. 99, no. 12, pp. 7821-7826, 2002.

[44] P. Holme and B. J. Kim, "Growing scale-free networks with tunable clustering," Physical Review E, vol. 65, no. 2, Article ID 026107, 2002.

[45] M. Small, Y. Li, T. Stemler, and K. Judd, "Growing optimal scale-free networks via likelihood," Physical Review E, vol. 91, no. 4, Article ID 042801, 2015.

[46] Z. Liu, Y.-C. Lai, and N. Ye, "Propagation and immunization of infection on general networks with both homogeneous and heterogeneous components," Physical Review E, vol. 67, no. 3, Article ID 031911, 2003.

[47] M. Molloy and B. Reed, "A critical point for random graphs with a given degree sequence," Random Structures \& Algorithms, vol. 6, no. 2-3, pp. 161-180, 1995.

[48] M. Catanzaro, M. Boguñá, and R. Pastor-Satorras, "Generation of uncorrelated random scale-free networks," Physical Review E, vol. 71, no. 2, Article ID 027103, 2005.

[49] M. Boguná, R. Pastor-Satorras, and A. Vespignani, "Cut-offs and finite size effects in scale-free networks," The European Physical Journal B-Condensed Matter and Complex Systems, vol. 38, no. 2, pp. 205-209, 2004.

[50] S. C. Ferreira, C. Castellano, and R. Pastor-Satorras, "Epidemic thresholds of the susceptible-infected-susceptible model on networks: a comparison of numerical and theoretical results," Physical Review E, vol. 86, no. 4, Article ID 041125, 2012.

[51] X. Chen, C. Yang, L. Zhong, and M. Tang, "Crossover phenomena of percolation transition in evolution networks with hybrid attachment," Chaos: An Interdisciplinary Journal of Nonlinear Science, vol. 26, no. 8, Article ID 083114, 2016.

[52] S. Boccaletti, V. Latora, Y. Moreno, M. Chavez, and D.-U. Hwang, "Complex networks: structure and dynamics," Physics Reports, vol. 424, no. 4-5, pp. 175-308, 2006.

[53] M. Newman, Networks: An Introduction, Oxford University Press, Oxford, UK, 2010.

[54] R. Pastor-Satorras and A. Vespignani, "Epidemic spreading in scale-free networks," Physical Review Letters, vol. 86, no. 14, pp. 3200-3203, 2001.

[55] http://finance.sina.com.cn/china/gncj/2020-03-26/dociimxxsth 1817404 .shtml. 